\title{
Variabilidade espacial de atributos químicos do solo sob cafeeiro Conilon: relação com textura, matéria orgânica e relevo
}

\author{
Diego Lang Burak (*); Renato Ribeiro Passos; Felipe Vaz Andrade \\ Universidade Federal do Espírito Santo, Centro de Ciências Agrárias, Departamento de Produção Vegetal, Alto Universitário, s/n, \\ 29500-000 Alegre (ES), Brasil. \\ (*) Autor correspondente: diego.burak@ufes.br
}

Recebido: 20/mar./2012; Aceito: 6/dez./2012

\begin{abstract}
Resumo
Atributos químicos do solo têm relação com outros atributos, inclusive os geomorfológicos, constituindo fatores que controlam sua variabilidade espacial. Dentro desta premissa, delineou-se o objetivo deste trabalho: avaliar a variabilidade dos atributos químicos do solo e sua inter-relação com as frações granulométricas e componentes da matéria orgânica do solo, contextualizando a influência do relevo, utilizando-se a geoestatística e a análise de componentes principais (ACP). A variabilidade espacial dos atributos químicos em menor profundidade foi mais influenciada pelo relevo. Pela ACP, atributos relacionados com a reação do solo $(\mathrm{pH}, \mathrm{Ca}, \mathrm{Mg}, \mathrm{Al}, \mathrm{m}$ e V) contribuíram com o primeiro componente principal (CP1) nas duas profundidades. Contudo, somente o CP1 na profundidade de 0,0-0,1 m foi correlacionado com o relevo: maiores altitudes e menores declividades favoreceram o maior teor da fração argila e menor teor de areia grossa, aumentando a retenção de cátions básicos no solo. Substâncias húmicas da matéria orgânica tiveram fraca influência na variabilidade dos atributos químicos, ressalvando a maior relação entre Ca e Mg com ácidos húmicos em locais de textura mais arenosa. Zonas homogêneas e correlatas com atributos geomorfológicos nas duas profundidades foram melhores obtidas para os teores de K, em comparação aos demais atributos. Dessa forma, o uso da análise espacial para segmentação da paisagem em regiões homogêneas visando otimizar o manejo da adubação depende não somente da topografia, mas também dos atributos químicos e da profundidade de amostragem.
\end{abstract}

Palavras-chave: fertilidade do solo, atributos do solo, atributos geomorfológicos, análise geoestatística, análise de componentes principais.

\section{Spatial variability of soil chemical attributes in Conilon coffee plantation: relationships with soil texture, organic matter and relief}

\begin{abstract}
Chemical properties are related to other soil attributes, including geomorphology, and constitute one of the factors that control spatial variability. The objective of this work was to evaluate the variability of soil chemistry and how it is interrelated with granulometric size fractions and organic soil components. Geostatistics and Principal Component Analysis (PCA) were used to describe the influence of topography. Spatial variability was more influenced by topography at lower depths. The PCA technique showed that attributes associated with soil reactions $(\mathrm{pH}, \mathrm{Ca}, \mathrm{Mg}, \mathrm{Al}, \mathrm{V}$ and $\mathrm{m}$ ) contributed to the first principal component (PC1) at the two studied depths. Only PC1 at the 0.0-0.1 m depth was, however, correlated to topography; higher altitudes and smaller slopes favored higher clay content and lower coarse sand proportion, thus resulting in increased retention of cations in the soil. The humic substances of organic matter have low influence on the variability of the chemical attributes, with the exception of $\mathrm{Ca}$ and $\mathrm{Mg}$ that are well related to humic acids in sites that are richer in sand. Homogeneous areas correlated with geomorphologic attributes was been describe for the $\mathrm{K}$ concentrations compared to other attributes at both depths. Thus, the use of spatial analysis for landscape stratification in homogeneous regions for the purpose of fertilizer management depends not only on topography, but on the chemical attributes evaluated and on sample depth.
\end{abstract}

Key words: soil fertility, soil attributes, geomorphologic attributes, geostatistical analysis, principal component analysis. 


\section{INTRODUÇÃO}

Inúmeros fatores, ou de origem natural ou antrópica, geram a variabilidade espacial dos atributos químicos do solo. O estudo desses fatores, também ditos intrínsecos (fatores de formação do solo - clima, material de origem, relevo, organismo e tempo) ou extrínsecos (atividades de manejo - adubações, aração e gradagem, rotação de culturas, entre outros), torna-se importante na estratificação de uma área em zonas homogêneas que demandam um manejo específico contribuindo, por exemplo, na aplicação racional de adubos e corretivos, prática inserida no contexto do manejo sustentável dos recursos agrícolas (Cambardella et al., 1994). Contudo, distinção das diferentes fontes de variação associadas aos fatores intrínsecos e extrínsecos é dificultada visto que elas são diversas e atuam simultaneamente no solo.

A variabilidade de atributos do solo em função dos fatores de formação do solo, em menor escala como a do segmento de uma paisagem (terço superior, médio e inferior), é influenciada predominantemente pela dinâmica da água, que tem forte relação com relevo. Esse fato gera fluxos de materiais constituindo os fatores ambientais de maior importância na variabilidade espacial de atributos do solo (Ceddia et al., 2009). Com o uso do solo, a variabilidade espacial naturalmente encontrada pode ser modificada com o efeito do preparo do solo, efeitos residuais de adubos e corretivos, sistemas de manejo adotados, forma de aplicação de insumos, entre outros fatores que podem em algumas situações tanto aumentar a heterogeneidade do solo como diminuí-la (CarvalHo et al., 2003, Cavalcante et al., 2007; Vieira et al., 2011).

$\mathrm{Na}$ análise simultânea de muitas informaçôes, a estatística multivariada torna-se a melhor ferramenta, possibilitando obter informaçōes e interpretações que poderiam não ser perceptíveis com o uso da análise estatística univariada (Cruz e Regazzi, 2001). Estudos na ciência do solo, nos quais muitas informaçōes necessitam ser interpretadas, adotaram a estatística multivariada e a integraram com a estatística espacial (FACCHINELli et al., 2001; BorU゚VKA et al., 2007). Nesse contexto, para compreensão da relaçáo do relevo com os diversos fatores responsáveis pela variabilidade dos atributos do solo, a análise espacial tem sido feita, por exemplo, com componentes principais em conjunto com atributos geomorfológicos (Sun et al., 2003; BorŮvKA et al., 2007). Essa relação se torna particularmente importante em cultivos de café sobre relevo ondulado, visto a ação diferenciada da dinâmica da água em camadas mais superficiais do solo. Além do fato da maior concentração de raízes do cafeeiro Conilon localizar-se nas camadas superficiais do solo (aproximadamente $50 \%$ na camada de $0,0-0,1 \mathrm{~m}$ e mais de $65 \%$ nas camadas de $0,0-0,2 \mathrm{~m}$ ) em variedades propagadas por estaca e sementes (PARTELLI et al., 2006).

O presente trabalho examinou a hipótese de que atributos químicos do solo se inter-relacionam com os demais atributos, inclusive geomorfológicos, constituindo fatores que influenciam a distribuição espacial e a formaçáo de zonas homogêneas de nutrientes. Para tanto, avaliou-se a variabilidade espacial de atributos químicos na profundidade de $0,0-0,1$ e $0,1-0,2 \mathrm{~m}$ do solo, assim como sua inter-relação com fraçôes granulométricas e componentes da matéria orgânica. Adicionalmente, avaliou-se a influência da declividade, altitude e fluxo acumulado do escoamento superficial. Utilizaram-se como ferramentas a geoestatistica e a análise de componentes principais.

\section{MATERIAL E MÉTODOS}

A área de estudo localiza-se na Fazenda Experimental Bananal do Norte, Cachoeiro de Itapemirim (ES), Brasil (204' $17,43^{\prime \prime} S$ a $20^{\circ} 45^{\prime} 17,73^{\prime \prime} S$; $41^{\circ} 17^{\prime} 11,26^{\prime \prime O}$ a $\left.41^{\circ} 17^{\prime} 6,62^{\prime \prime} \mathrm{O}\right)$. $\mathrm{Na}$ área há lavoura de café da espécie Coffea canephora Pierre, var. Robusta Tropical 'Emcaper 8151' em uma extensão de 0,6 ha desde 2000. O espaçamento da cultura é de 2,9x0,9 m.

O manejo da adubação é feito anualmente com aplicação de 130 gramas por planta do formulado 20-0-20 (uréia e cloreto de potássio) e uma aplicação de $80 \mathrm{~g}$ por planta de superfosfato simples. Após a adubação, é realizada a poda de produção e os restos vegetais da poda sáo distribuídos sobre o solo. A calagem é realizada por planta, anualmente, no período de agosto a outubro (após a colheita), sendo a dose calculada com base na análise de solo e na recomendação para o Estado do Espírito Santo (Prezotti et al., 2007). Esta mesma forma de manejo é realizada, anualmente, desde 2006, com os mesmos períodos de colheita, adubação e poda. O solo foi classificado como Latossolo Vermelho-Amarelo distrófico textura argilosa com relevo regional ondulado (8 a 20\% de declividade).

A amostragem para avaliação dos atributos químicos foi realizada com a coleta de quatro amostras simples com auxílio de pá reta no entorno de uma planta de café compondo 109 amostras compostas georreferenciadas na profundidade de $0,0-0,1$ e de $0,1-0,2 \mathrm{~m}$. A grade de amostragem foi do tipo irregular com 2,9 a $10 \mathrm{~m}$ entre pontos.

Após a coleta, secagem e peneiramento para obtenção da terra fina seca ao ar, as amostras foram caracterizadas quanto a: $\mathrm{pH}$ em água, utilizando as proporçôes 1:2,5 (v/v) de solo:solução; cálcio, magnésio e alumínio extraídos por soluçáo de $\mathrm{KCl} 1 \mathrm{~mol} \mathrm{~L}^{-1}$ e os teores determinados por espectrometria de absorção atômica; e potássio e fósforo extraídos por Mehlich-1 e determinados, respectivamente, por fotometria de chama e colorimetria (Claessen, 1997). Para determinação da acidez potencial $(\mathrm{H}+\mathrm{Al})$, utilizou-se o extrator acetato de cálcio $0,5 \mathrm{~mol} \mathrm{~L}^{-1}$. Com base nessas determinaçóes, foram calculados: capacidade de troca de cátions (CTC) efetiva; soma de bases (SB), saturação por bases (V) e saturação por alumínio (m). Os teores de areia grossa, areia fina, silte e argila foram 
determinados conforme Claessen (1997) com modificaçôes. Os teores de carbono orgânico total (COT) foram determinados em amostras trituradas e passadas em peneira de $0,210 \mathrm{~mm}$ pela oxidação via úmida, utilizando-se solução de $\mathrm{K}_{2} \mathrm{Cr}_{2} \mathrm{O}_{7}$ em meio ácido e aquecimento externo (Yeomans e Bremner, 1988). O fracionamento químico das substâncias húmicas foi realizado com base nas características de solubilidade diferencial, obtendo-se as seguintes fraçôes: ácidos fúlvicos (CAF) — solúveis em ácido e em álcali; ácidos húmicos $(\mathrm{CAH})$ - solúveis em álcali e insolúveis em ácido; e huminas ( $\mathrm{CHU})$ - insolúveis em ácido e em álcali, conforme descrito em Canellas et al. (2000). A determinação do C orgânico das fraçôes ácidos fúlvicos e ácidos húmicos, obtidas na extração, foi feita utilizando-se o mesmo método do COT.

Para melhor visualização das formas do relevo na área de estudo foram avaliados a declividade e o fluxo acumulado do escoamento superficial (FAES), obtido a partir de Modelo Digital de Elevação (Figura 1). Os valores atribuídos aos pixels foram obtidos dos planos de informação em formato raster com resolução de $2 \mathrm{~m}$ (declividade e FAES), os quais foram avaliados junto à altitude gerando informaçôes quantitativas (atributos geomorfológicos) dos pontos georreferenciados.

Com os dados dos atributos químicos e físicos avaliados realizaram-se análises descritivas, análises de correlação de Pearson e análises de componentes principais (ACP). Para a ACP, foram retiradas a CTC e SB pela alta correlação com $\mathrm{Ca}$ e $\mathrm{Mg}$, evitando a redundância de informaçôes, assim como as variáveis $\mathrm{H}+\mathrm{Al}$, teores de silte e teores de areia fina, por ter maiores autovetores associados aos autovalores de menor magnitude em análises prévias (Cruz e Regazzi, 2001). Na ACP, fatores principais (componentes principais) que respondem pela maior parte da variabilidade de todas as variáveis são identificados, decompondo-se a matriz de correlação ou covariância (Cruz e Regazzi, 2001), sendo no presente estudo utilizada a matriz de correlação. A contribuição (loadings) dos atributos relacionados aos componentes principais (correlação entre atributos avaliados e os componentes principais) foi avaliada conforme o critério de seleção (CS) estabelecido por Ovalles e Collins (1988):

\section{$\mathrm{CS}=0,5 /(\mathrm{CP} \text { eigenvalue })^{1 / 2}$}

Em que: CS é o valor do critério de seleção e o CP eigenvalue é o autovalor do componente principal. Dessa forma, o CS (Equação 1) tem sido utilizado para estabelecer valores partir dos quais começa a existir uma contribuição da variável na variação explicada pelo componente principal (Wairegi e Asten, 2012).

A variabilidade espacial dos atributos químicos e físicos do solo, assim como dos componentes principais, foi caracterizada por semivariogramas; por náo ter sido constatada a anisotropia dos dados, as semivariâncias médias nas diferentes direçôes foram utilizadas (isotropia). Para interpolação, foi utilizada a krigagem ordinária. Detalhes da teoria geoestatística podem ser visualizados em Vieira (2000); Webster e Oliver (2007). Para a seleção dos semivariogamas, consideraram-se: os maiores valores de $\mathrm{R}^{2}$; menores valores de soma do quadrado do resíduo (dados não apresentados); maior coeficiente de correlaçáo entre dados estimados pela validação cruzada e dados reais; e, valores mais próximos a 1 e a zero para a variância e média padronizada da validação cruzada (VIEIRA, 2000; Webster e Oliver, 2007).

As análises estatísticas descritivas, correlaçôes de Pearson e a análise de componentes principais foram feitas com auxílio do software Statistica 6.0 ${ }^{\circledR}$. A análise geoestatística, bem como a interpolação, foram realizadas com (a) ALTITUDE

metros

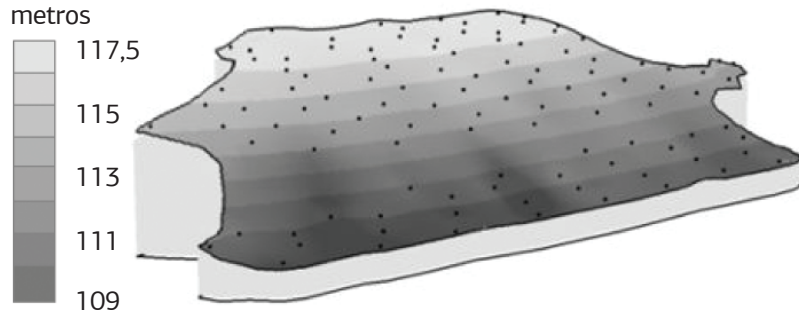

(b) DECLIVIDADE

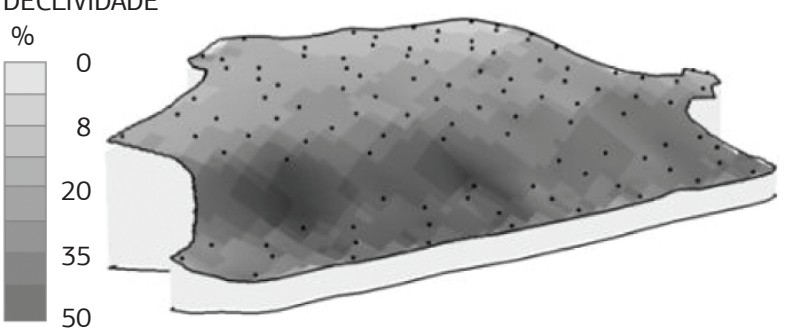

(c) FAES
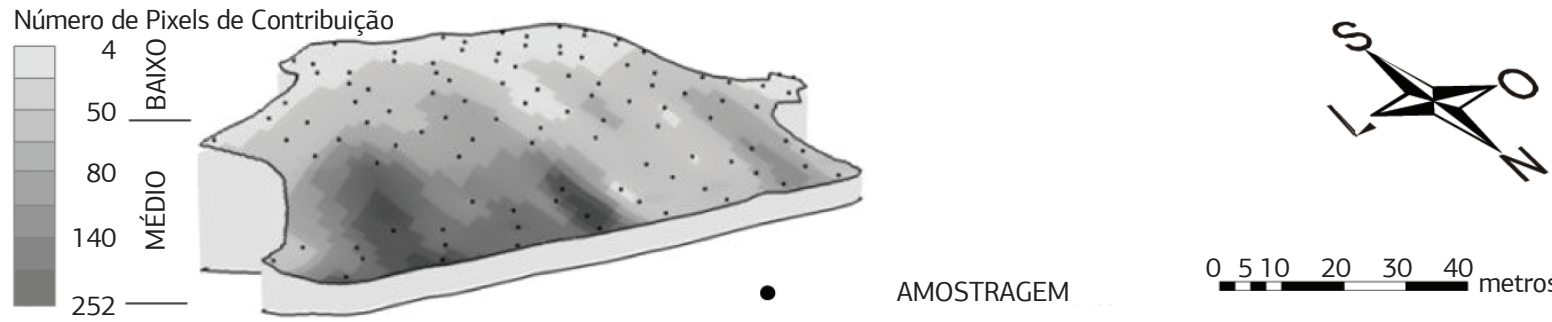

AMOSTRAGEM

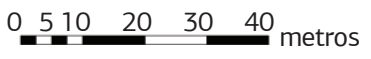

Figura 1. Modelo digital de elevaçáo (a), declividade (b), e fluxo acumulado do escoamento superficial da área de estudo (FAES), separado em classes de baixo e médio fluxo (c). 
software geoestatístico GS+, versão 7.0 (GAMma Design SOFTWARE, 2004) e os mapas confeccionados a partir de recursos oferecidos pelo software ArcGIS 9.2 Environmental Systems Research Institute ${ }^{\circledR}$.

\section{RESULTADOS E DISCUSSÃO}

As estatísticas descritivas dos atributos na profundidade de $0,0-0,1$ e $0,1-0,2$ m são apresentadas nas tabelas 1 e 2 . A partir da média ( $\mathrm{n}=109$ pontos) pode-se inferir que o solo possui alto nível de fertilidade, uma vez que os teores dos elementos nutrientes estáo entre as classes de alta (potássio e fósforo) e média (cálcio e magnésio) fertilidade para o Estado do Espírito Santo, segundo Prezzotti et al. (2007).

Interpretando-se a variaçáo dos dados em torno da média, pode-se constatar o menor coeficiente de variação para os atributos físicos em comparação aos atributos químicos e componentes relacionados à matéria orgânica como resultado da maior dinâmica desses últimos e, ou, efeitos residuais de adubação/calagem e

Tabela 1. Sumário da estatística descritiva dos atributos ${ }^{(1)}$ do solo na profundidade de $0,0-0,1 \mathrm{~m}$

\begin{tabular}{|c|c|c|c|c|c|c|}
\hline Variável & Média+Desvio-padrão & Mín & Máx & CV\% & Cs & Ck \\
\hline $\mathrm{pH}$ & $4,91 \pm 0,36$ & 4,00 & 5,94 & 7 & 0,15 & 0,16 \\
\hline$P\left(\mathrm{mg} \mathrm{dm}^{-3}\right)$ & $25,14 \pm 14,94$ & 5,28 & 66,15 & 59 & 1,28 & 2,36 \\
\hline $\mathrm{K}\left(\mathrm{mg} \mathrm{dm} \mathrm{m}^{-3}\right)$ & $194,75 \pm 115,12$ & 39,00 & 854,00 & 60 & 2,92 & 14,00 \\
\hline $\mathrm{Al}\left(\mathrm{cmol}_{\mathrm{c}} \mathrm{dm}^{-3}\right)$ & $0,10 \pm 0,08$ & 0,01 & 0,51 & 83 & 1,89 & 5,37 \\
\hline $\mathrm{Ca}\left(\mathrm{cmol}_{\mathrm{c}} \mathrm{dm}^{-3}\right)$ & $2,70 \pm 0,73$ & 0,86 & 4,80 & 27 & 0,12 & 0,28 \\
\hline $\mathrm{Mg}\left(\mathrm{cmol}_{\mathrm{c}} \mathrm{dm}^{-3}\right)$ & $0,93 \pm 0,28$ & 0,32 & 1,76 & 29 & 0,36 & 0,07 \\
\hline $\mathrm{H}+\mathrm{Al}\left(\mathrm{cmol}_{\mathrm{c}} \mathrm{dm}^{-3}\right)$ & $4,13 \pm 1,05$ & 1,37 & 6,76 & 25 & 0,09 & 0,07 \\
\hline $\mathrm{CTC}_{\text {efetiva }}\left(\mathrm{cmol}_{\mathrm{c}} \mathrm{dm}^{-3}\right)$ & $8,64 \pm 1,17$ & 6,01 & 11,67 & 14 & 0,28 & 0,07 \\
\hline V (\%) & $58,69 \pm 6,55$ & 43,19 & 77,79 & 11 & 0,20 & 0,01 \\
\hline m (\%) & $1,31 \pm 1,25$ & 0,07 & 7,60 & 96 & 2,30 & 7,15 \\
\hline $\operatorname{COT}\left(\mathrm{g} \mathrm{kg}^{-1}\right)$ & $13,9 \pm 4,9$ & 6,51 & 27,2 & 35 & 0,72 & 0,54 \\
\hline CAF $\left(\mathrm{g} \mathrm{kg}^{-1}\right)$ & $2,55 \pm 0,36$ & 1,57 & 3,33 & 14 & $-0,25$ & 0,08 \\
\hline $\mathrm{CAH}\left(\mathrm{g} \mathrm{kg}^{-1}\right)$ & $2,80 \pm 0,61$ & 1,43 & 4,23 & 22 & $-0,04$ & $-0,16$ \\
\hline $\mathrm{CHU}\left(\mathrm{g} \mathrm{kg}^{-1}\right)$ & $8,68 \pm 1,51$ & 4,94 & 11,83 & 17 & $-0,49$ & 0,00 \\
\hline $\operatorname{ArG}($ dag kg-1) & $32,14 \pm 4,50$ & 21,58 & 43,63 & 14 & 0,24 & $-0,01$ \\
\hline $\operatorname{ArF}\left(\right.$ dag kg $\left.{ }^{-1}\right)$ & $11,85 \pm 1,67$ & 8,42 & 16,92 & 14 & 0,33 & $-0,07$ \\
\hline Sil (dag kg-1) & $5,45 \pm 2,97$ & 0,42 & 13,84 & 55 & 1,17 & 0,85 \\
\hline $\operatorname{Arg}($ dag kg-1) & $50,59 \pm 4,95$ & 35,82 & 62,15 & 10 & $-0,44$ & 0,70 \\
\hline
\end{tabular}

(1) COT: carbono orgânico total; CAF: carbono orgânico da fração ácidos fúlvicos; CAH: carbono orgânico da fração ácidos húmicos; CHU: carbono orgânico da fração humina; ArG: areia grossa; Sil: silte; ArF: areia fina; Arg: argila; Min: mínimo; Max: máximo; CV\%: coeficiente de variação; Cs: coeficiente de assimetria; Ck: coeficiente de curtose.

Tabela 2. Sumário das estatísticas descritivas dos atributos ${ }^{(1)}$ do solo na profundidade de $10-20 \mathrm{~m}$

\begin{tabular}{|c|c|c|c|c|c|c|}
\hline Variável & Média+Desvio-padrão & Mín & Máx & CV\% & Cs & Ck \\
\hline $\mathrm{pH}$ & $4,65 \pm 0,35$ & 3,80 & 5,70 & 7 & 0,46 & 0,34 \\
\hline$P\left(m g d^{-3}\right)$ & $38,48 \pm 23,42$ & 6,10 & 150,46 & 61 & 1,74 & 5,32 \\
\hline $\mathrm{K}\left(\mathrm{mg} \mathrm{dm^{-3 } )}\right.$ & $233,60 \pm 132,91$ & 34,00 & 787,00 & 56 & 1,74 & 1,86 \\
\hline $\mathrm{Al}\left(\mathrm{cmol}_{\mathrm{c}} \mathrm{dm}^{-3}\right)$ & $0,19 \pm 0,15$ & 0,01 & 0,63 & 81 & 1,00 & 0,13 \\
\hline $\mathrm{Ca}\left(\mathrm{cmol}_{\mathrm{c}} \mathrm{dm}^{-3}\right)$ & $1,96 \pm 0,61$ & 0,78 & 3,63 & 30 & 0,39 & $-0,37$ \\
\hline $\mathrm{Mg}\left(\mathrm{cmol}_{\mathrm{c}} \mathrm{dm}^{-3}\right)$ & $0,66 \pm 0,25$ & 0,25 & 1,56 & 36 & 0,66 & 0,78 \\
\hline $\mathrm{H}+\mathrm{Al}\left(\mathrm{cmol}_{\mathrm{c}} \mathrm{dm}^{-3}\right)$ & $4,97 \pm 1,47$ & 2,15 & 14,03 & 29 & 2,08 & 12,33 \\
\hline CTC ${ }_{\text {efetiva }}\left(\mathrm{cmol}_{\mathrm{c}} \mathrm{dm}^{-3}\right)$ & $3,40 \pm 0,92$ & 1,89 & 5,92 & 27 & 0,56 & $-0,30$ \\
\hline V (\%) & $39,56 \pm 10,66$ & 16,01 & 66,93 & 26 & 0,34 & $-0,04$ \\
\hline m (\%) & $6,46 \pm 6,10$ & 0,15 & 28,48 & 94 & 1,37 & 1,56 \\
\hline $\mathrm{COT}\left(\mathrm{g} \mathrm{kg}^{-1}\right)$ & $13,8 \pm 4,32$ & 3,95 & 27,23 & 31 & 0,59 & 1,62 \\
\hline CAF $\left(\mathrm{g} \mathrm{kg}^{-1}\right)$ & $2,43 \pm 0,37$ & 1,54 & 3,48 & 15 & 0,47 & 0,50 \\
\hline CAH $\left(\mathrm{g} \mathrm{kg}^{-1}\right)$ & $2,68 \pm 0,96$ & 1,05 & 5,59 & 35 & 0,27 & $-0,30$ \\
\hline $\mathrm{CHU}\left(\mathrm{g} \mathrm{kg}^{-1}\right)$ & $6,99 \pm 1,11$ & 4,22 & 9,90 & 15 & $-0,26$ & 0,34 \\
\hline $\operatorname{ArGR}\left(\operatorname{dag} \mathrm{kg}^{-1}\right)$ & $30,10 \pm 4,70$ & 16,30 & 41,03 & 15 & $-0,21$ & 0,44 \\
\hline $\operatorname{ArF}\left(\right.$ dag $\left.\mathrm{kg}^{-1}\right)$ & $11,60 \pm 1,41$ & 7,50 & 15,01 & 12 & $-0,02$ & 0,08 \\
\hline Sil (dag kg $\left.{ }^{-1}\right)$ & $5,81 \pm 4,65$ & 0,40 & 26,81 & 79 & 2,30 & 6,09 \\
\hline $\operatorname{Arg}\left(\operatorname{dag} \mathrm{kg}^{-1}\right)$ & $52,40 \pm 6,71$ & 30,36 & 73,36 & 12 & $-0,38$ & 1,57 \\
\hline
\end{tabular}

(') COT: carbono orgânico total; CAF: carbono orgânico da fração ácidos fúlvicos; CAH: carbono orgânico da fração ácidos húmicos; CHU: carbono orgânico da fraçāo humina; ArG: areia grossa; Sil: silte; ArF: areia fina; Arg: argila; Min: mínimo; Max: máximo; CV\%: coeficiente de variação; Cs: coeficiente de assimetria; Ck: coeficiente de curtose. 
do manejo dos restos vegetais, segundo outros autores (Carvalho et al., 2003; Sun et al., 2003; Cavalcante et al., 2007; Vieira et al, 2011). Destacam-se os valores de $\mathrm{P}, \mathrm{K}$ e Al considerados em geral com alto coeficiente de variação (Tabelas 1 e 2). Essas variáveis tiveram acentuada assimetria, sendo os valores extremos agrupados espacialmente ao serem avaliados pelo Índice de Moran's (Zhang e McGrath, 2004). Nesse contexto, os valores de $\mathrm{P}, \mathrm{K} \mathrm{e} \mathrm{Al}$ necessitaram de transformaçáo logarítmica ao invés do descarte de dados, tornando os semivariogramas mais robustos e as estimativas mais confiáveis da krigagem (Webster e Oliver, 2007). Estes dados transformados (P, $\mathrm{K}$ e Al) foram utilizados na análise de componentes principais que, embora formalmente não exija distribuição normal, torna-se desejável a utilização de dados com menor assimetria (JAMES e McCulloch, 1990).

$\mathrm{Na}$ maioria das variáveis houve dependência espacial, com exceção do $\mathrm{P}$ e $\mathrm{Mg}$ na profundidade de $0,1-0,2 \mathrm{~m}$ em que ocorreu efeito pepita puro (EPP), significando que para esses atributos químicos a variabilidade aleatória predomina sobre a variabilidade espacialmente estruturada (Tabela 3). Carvalho et al. (2003) verificaram dependência espacial somente na camada de $0-5 \mathrm{~m}$ para o $\mathrm{P}$ em cultivo de videira no Sul do Brasil.

Os modelos ajustados foram predominantemente os exponenciais. Um elevado efeito pepita pode ser indicativo de erros de amostragem devido à variaçáo localizada, erros de análises, ou esquema de amostragem não suficiente para detecção da estrutura espacial dos dados (Vieira, 2000; Webster e Oliver, 2007). O efeito pepita, comparados por meio do grau de dependência espacial (Tabela 3), em geral, foram maiores na profundidade de 0,0-0,1 m (CAmbardella et al., 1994).

Valores de alcance para todos os atributos químicos estudados foram maiores em superfície (Tabela 3), sugerindo que a continuidade da dependência espacial é maior pelo efeito de carreamento via escoamento superficial por influência do relevo (fator intrínseco). Maiores valores de alcance para alguns atributos avaliados em camadas mais superficiais de solo têm sido constatados na literatura (SouzA et al., 2006), assim como efeito do manejo do solo, preparo convencional, por exemplo, diminuindo os valores do alcance (CAvalCante et al., 2007). Deve-se levar também em consideração a mobilidade dos elementos no solo: menores alcances para o $\mathrm{P}$ e maiores para o $\mathrm{K}, \mathrm{pH}$ e soma de bases sugerem o resultado da maior mobilidade de $\mathrm{K}$ e das demais bases trocáveis no solo como observado também por SouzA et al. (2006). A ordem decrescente do alcance no presente estudo foi de: $\mathrm{pH}>\mathrm{K}>\mathrm{Al}>\mathrm{Ca}>\mathrm{Mg} \approx \mathrm{P}$ sugerindo que, em geral, elementos menos móveis após seu aporte pela fertilização ou aplicação de corretivos tiveram menor alcance. Sun et al. (2003) verificaram diminuição do alcance e aumento do efeito pepita sob influência do manejo no solo (fator extrínseco), sendo tal comportamento mais importante para a matéria orgânica e $\mathrm{P}$, em comparação ao $\mathrm{K}$ e $\mathrm{pH}$, que tiveram maiores valores de alcance.

Por meio da interpolação dos dados pela krigagem, pode-se observar que na profundidade de $0,0-0,1 \mathrm{~m}$ maiores teores de $\mathrm{Ca}, \mathrm{Mg}$ e $\mathrm{K}$ predominam nos locais em maior altitude, no terço superior da área estudada (Figura 2). Já em menor altitude, no terço médio do segmento estudado, são observados maiores teores de $\mathrm{Al}$ e menores valores de $\mathrm{pH}$. Pelo mapa de distribuiçâao espacial, pouca relação foi observada entre $\mathrm{P}$ e relevo na profundidade de $0,0-0,1 \mathrm{~m}$. Na profundidade de $0,1-0,2 \mathrm{~m}$, foi menos evidenciada a tendência de maiores valores de $\mathrm{Ca}, \mathrm{K}, \mathrm{Al}$ e $\mathrm{pH}$ em maior altitude.

A ACP possibilita melhor avaliaçáo de grupos de atributos inter-relacionadas no solo a partir da sua interpretação, distribuição espacial e correlação com atributos geomorfológicos. Na ACP, para as variáveis na profundidade de $0,0-0,1 \mathrm{~m}$, foram avaliados os quatro primeiros

Tabela 3. Modelos teóricos e parâmetros estimados dos semivariogramas experimentais ajustados aos valores dos atributos químicos do solo nas profundidades de 0,0-0,1 e 0,1-0,2 m e a estatística da validação cruzada

\begin{tabular}{|c|c|c|c|c|c|c|c|c|c|c|}
\hline \multirow{2}{*}{ Var. } & \multirow{2}{*}{$\begin{array}{l}\text { Profundidade } \\
\text { (m) }\end{array}$} & \multirow{2}{*}{ Modelo(1) } & \multicolumn{5}{|c|}{ Parâmetros ${ }^{(2)}$} & \multicolumn{3}{|c|}{ Validação Cruzada(4) } \\
\hline & & & $C_{0}$ & $C_{0}+C_{1}$ & $C_{0} /\left(C_{0}+C_{1}\right)^{(3)}$ & A & $\mathbf{R}^{2}$ & Variância & Média & $\mathbf{r}$ \\
\hline \multirow{2}{*}{$P^{(5)}$} & $0,0-0,1$ & EXP & 0,03 & 0,23 & 13 & 11,2 & 0,94 & 1,01 & 0,001 & $0,26^{*}$ \\
\hline & $0,1-0,2$ & EPP & - & - & - & - & - & - & - & - \\
\hline \multirow{2}{*}{$K^{(5)}$} & $0,0-0,1$ & EXP & 0,48 & 0,68 & 71 & 37,0 & 0,70 & 0,98 & 0,013 & $0,34^{* *}$ \\
\hline & $0,1-0,2$ & EXP & 0,11 & 0,26 & 42 & 17,5 & 0,82 & 1,06 & 0,012 & $0,27^{*}$ \\
\hline \multirow{2}{*}{$\mathrm{pH}$} & $0,0-0,1$ & EXP & 0,07 & 0,14 & 50 & 39,9 & 0,63 & 1,02 & 0,001 & $0,32 * *$ \\
\hline & $0,1-0,2$ & EXP & 0,02 & 0,13 & 15 & 15,7 & 0,98 & 0,99 & 0,002 & $0,28^{* *}$ \\
\hline \multirow{2}{*}{$\mathrm{Ca}$} & $0,0-0,1$ & EXP & 0,05 & 0,46 & 11 & 12,9 & 0,58 & 0,97 & 0,017 & $0,28^{* *}$ \\
\hline & $0,1-0,2$ & GAUS & 0,01 & 0,34 & 3 & 6,5 & 0,88 & 1,02 & 0,006 & $0,25^{*}$ \\
\hline \multirow{2}{*}{$\mathrm{Mg}$} & $0,0-0,1$ & ESF & 0,01 & 0,06 & 17 & 10,5 & 0,87 & 1,01 & 0,001 & $0,22^{*}$ \\
\hline & $0,1-0,2$ & EPP & - & - & - & - & - & - & - & - \\
\hline \multirow{2}{*}{$\left.A\right|^{(5)}$} & $0,0-0,1$ & EXP & 0,54 & 0,87 & 62 & 28,7 & 0,68 & 0,99 & 0,003 & $0,37^{* *}$ \\
\hline & $0,1-0,2$ & GAUS & 0,05 & 0,85 & 6 & 8,7 & 0,95 & 0,97 & 0,001 & $0,41^{* *}$ \\
\hline
\end{tabular}

Var.: Variável; (') EXP: exponencial; GAUS: gaussiano; ESF: esférico; EPP: efeito pepita puro. $\left(^{2}\right)$ Co: efeito pepita; Co+C1: patamar; A: alcance; $\mathrm{R}^{2}$ : coeficiente de determinação do semivariograma. $\left({ }^{3}\right)$ grau de dependência espacial expressa em porcentagem $(<25 \%$ - forte dependência espacial, 25 a $75 \%$ - moderada dependência espacial e $>75 \%$ - fraca dependência espacial) (CAmbardella et al., 1994). $\left({ }^{4}\right)$ Validação cruzada feita com a média do erro reduzido e a variância do erro reduzido, e coeficiente de correlaçáo simples entre dados observados e estimado da validaçáo cruzada, sendo ** significando $\mathrm{p}<0,01 \mathrm{e}^{*}$ significando $\mathrm{p}<0,05$. $\left(^{5}\right)$ dados com transformação logarítmica. 
componentes que explicaram $69,5 \%$ da variabilidade total dos dados e que tiveram autovalores maiores que 1 (Tabela 4). Segundo Cruz e Regazzi (2001), em estudos de ACP almeja-se que $70 \%$ da variaçáo total acumulada sejam explicadas pelos dois primeiros componentes. Quando tal acumulação não ocorre, pode-se optar pela utilização dos primeiros componentes com autovalores maiores que 1 que é a variância média dos atributos quando uma matriz de correlação é utilizada. (a)

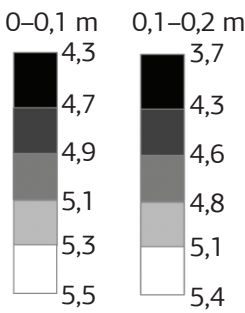

(c) $\mathrm{Ca}-\mathrm{cmol}_{\mathrm{c}} \mathrm{dm}^{3}$ $0-0,1 \mathrm{~m} \quad 0,1-0,2 \mathrm{~m}$
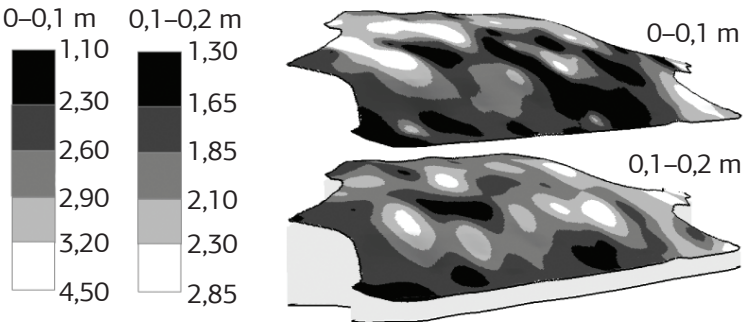

(e)

$\mathrm{K}-\mathrm{mg} \mathrm{dm} \mathrm{m}^{-3}$ $0-0,1 \mathrm{~m} \quad 0,1-0,2 \mathrm{~m}$
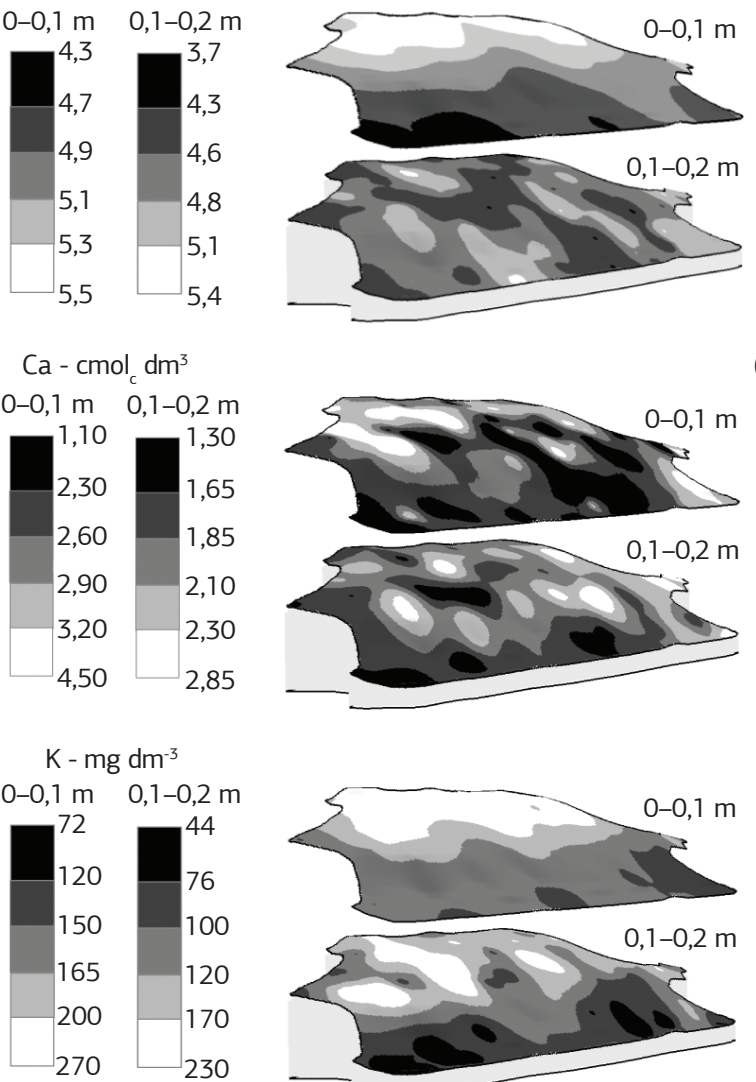

(d) $\mathrm{Mg}-\mathrm{Cmol}_{\mathrm{c}} \mathrm{dm}^{-3}$

0-0,1 m 0,1-0,2 m

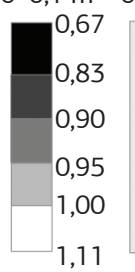

$0,66 \pm 0,25$ valor médio

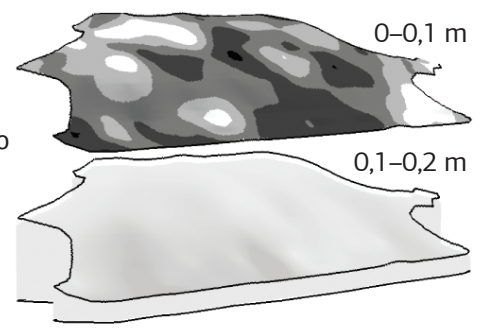

(f) $\quad P-m g d m^{-3}$
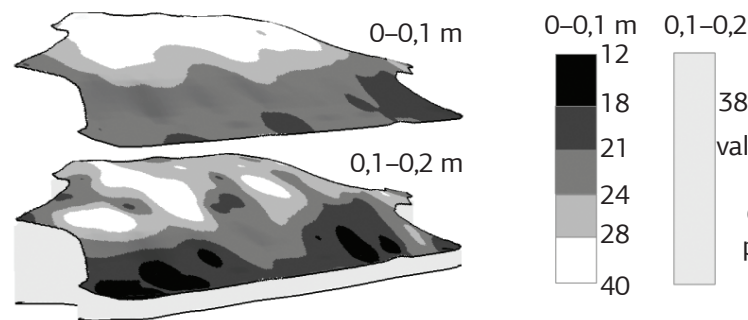

$38,5 \pm 23,4$ valor médio desvio padrão
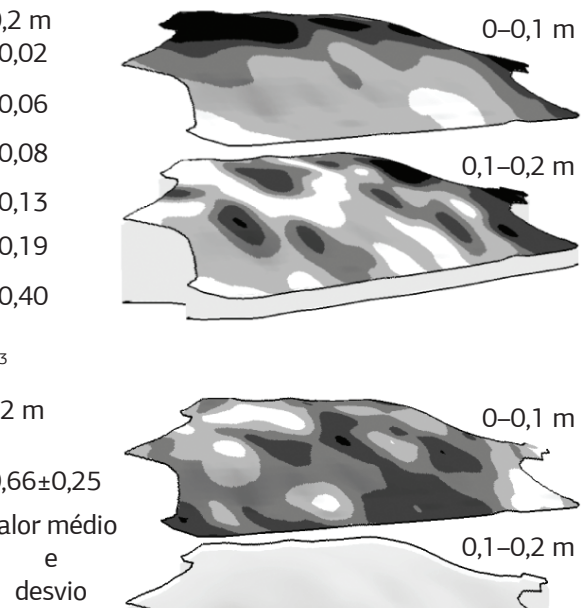
padrão

Figura 2. Distribuição espacial do $\mathrm{pH}$ (a) e dos teores de alumínio (b), cálcio (c), magnésio (d), potássio (e) e fósforo (f) nas profundidades de $0-0,1$ e $0,1-0,2 \mathrm{~m}$.

Tabela 4. Variância explicada pelos componentes principais e correlação( ${ }^{(1)}$ entre variáveis originais e componentes principais nas profundidades de $0,0-0,1$ e de $0,1-0,2 \mathrm{~m}$

\begin{tabular}{|c|c|c|c|c|c|c|c|c|}
\hline Componentes & CP 1 & CP 2 & CP 3 & CP 4 & CP 1 & CP 2 & CP 3 & CP 4 \\
\hline Autovalor & 4,77 & 2,77 & 1,20 & 1,00 & 4,38 & 2,13 & 1,25 & 1,15 \\
\hline Var. (\%) & 34,10 & 19,77 & 8,59 & 7,05 & 31,28 & 15,20 & 8,96 & 8,18 \\
\hline Var. Acum. (\%) & 34,10 & 53,86 & 62,45 & 69,50 & 31,28 & 46,48 & 55,44 & 63,62 \\
\hline Variáveis & \multicolumn{4}{|c|}{$0,0-0,1 \mathrm{~m}$} & \multicolumn{4}{|c|}{$0,1-0,2 \mathrm{~m}$} \\
\hline$P$ & 0,05 & 0,18 & $-0,17$ & $-0,22$ & 0,31 & 0,24 & 0,17 & $-0,29$ \\
\hline K & 0,36 & $-0,43$ & 0,07 & 0,17 & $-0,15$ & $-0,52$ & 0,02 & $-0,13$ \\
\hline $\mathrm{pH}$ & 0,75 & $-0,01$ & $-0,06$ & 0,15 & $-0,79$ & 0,13 & 0,03 & 0,13 \\
\hline Al & $-0,85$ & $-0,15$ & $-0,13$ & $-0,04$ & 0,87 & $-0,09$ & $-0,03$ & $-0,02$ \\
\hline $\mathrm{Ca}$ & 0,89 & 0,45 & 0,00 & $-0,02$ & $-0,79$ & 0,00 & 0,06 & $-0,17$ \\
\hline $\mathrm{Mg}$ & 0,58 & 0,32 & 0,09 & $-0,01$ & $-0,81$ & 0,05 & $-0,02$ & $-0,02$ \\
\hline V & 0,67 & 0,45 & 0,19 & 0,10 & $-0,85$ & $-0,13$ & $-0,01$ & $-0,08$ \\
\hline $\mathrm{m}$ & $-0,87$ & $-0,18$ & $-0,13$ & $-0,03$ & 0,92 & 0,05 & 0,00 & 0,05 \\
\hline COT & 0,36 & 0,21 & $-0,37$ & $-0,44$ & $-0,01$ & 0,10 & 0,73 & 0,14 \\
\hline CAF & 0,25 & $-0,10$ & $-0,66$ & 0,13 & $-0,09$ & 0,21 & $-0,08$ & 0,57 \\
\hline $\mathrm{CAH}$ & $-0,08$ & 0,36 & 0,16 & $-0,56$ & 0,05 & $-0,03$ & 0,30 & $-0,77$ \\
\hline $\mathrm{CHU}$ & 0,21 & 0,26 & $-0,41$ & $-0,51$ & $-0,05$ & 0,16 & 0,75 & $-0,01$ \\
\hline ArG & $-0,43$ & 0,60 & $-0,24$ & 0,34 & $-0,03$ & 0,84 & 0,11 & $-0,21$ \\
\hline Arg & 0,68 & $-0,61$ & 0,35 & $-0,24$ & 0,08 & $-0,82$ & $-0,07$ & 0,17 \\
\hline
\end{tabular}

(') Valores em negrito maiores que Critério de Seleçăo (Equação 1). 
No primeiro componente principal da profundidade de $0,0-0,1 \mathrm{~m}\left(\mathrm{CP} 1_{0,0-0,1}\right)$ explica-se $34,1 \%$ da variabilidade total dos dados e a elevada correlaçáo positiva com as variáveis $\mathrm{pH}$ e $\mathrm{Ca}$ e negativa com $\mathrm{Al}$ e $\mathrm{m}$ (Tabela 4). Menores correlaçóes positivas foram observadas entre $\mathrm{CP}_{0,0-0,1}$ e $\mathrm{K}, \mathrm{Mg}$, COT, CAF, CHU e argila, e negativas com areia grossa. A importância relativa ou influência de cada atributo químico em estudo sobre os componentes é dada pelas suas correlaçóes com os componentes principais. O grupo de maior contribuição da variação de $\mathrm{CP} 1_{0,0-0,1}(\mathrm{Ca}, \mathrm{Al}, \mathrm{m}$ e pH) identifica a variação na área inerente às reaçôes ácido-base no solo. $\mathrm{O}$ grupo das variáveis de menor contribuiçáo para o $\mathrm{CP} 1_{0,0-0,1}(\mathrm{~K}, \mathrm{Mg}$, COT, CAF, CHU e argila) pode ser interpretado como o efeito principalmente dos teores de argila e menor contribuição da matéria orgânica na geração de cargas e retenção de cátions aportados predominantemente via adubação.

$\mathrm{O}$ coeficiente de correlação de Pearson entre $\mathrm{CTC}_{\text {efeiva }} \mathrm{x}$ argila e $\mathrm{CTC}_{\text {efeiva }} \times \mathrm{COT}$ na profundidade entre $0,0-0,1 \mathrm{~m}$ foi de, respectivamente, 0,23 e 0,21 , indicando uma correlaçáo significativa $(\mathrm{p}<0,05)$ entre esses atributos, porém de fraca intensidade (SANTOS et al., 2012). $\mathrm{Na}$ literatura, tem sido registrado o efeito dos teores de argila e da matéria orgânica no aumento da CTC do solo (Dobbs et al., 2008). Com a retenção desses cátions básicos, locais com maiores valores de $\mathrm{Ca}$ e $\mathrm{pH}$ possuem menores teores de $\mathrm{Al}$, visto que a presença de cátions básicos aumenta o $\mathrm{pH}$ promovendo a precipitaçáo do $\mathrm{Al}$ em hidróxidos.

No segundo componente principal $\left(\mathrm{CP}_{0,0-0,1}\right)$, correspondendo a $19,77 \%$ da variância total dos dados, observa-se a contribuição da areia grossa, $\mathrm{Ca}, \mathrm{Mg}$, $\mathrm{V}$, COT e CAH, que apresentaram correlação positiva com CP2 $2_{0,0-0,1}$ destacando a correlação negativa da argila com CP2 $2_{0,0-0,1}$ (Tabela 4). O grupo de atributos relacionado ao $\mathrm{CP} 2_{0,0-0,1}$ (areia grossa, $\mathrm{Ca}, \mathrm{Mg}, \mathrm{V}$, COT e $\mathrm{CAH}$ ) sugerem a maior influência de componentes orgânicos, em relação aos minerais na retenção de bases, uma vez que indicam locais com menores teores de argila e maiores teores de areia grossa onde se sobressai a contribuição da matéria orgânica. A correlaçáo negativa do $\mathrm{K}$ com $\mathrm{CP} 2_{0,0-0,1}$ corrobora a idéia da maior influência da matéria orgânica como geradora de cargas no solo, visto a baixa afinidade do $\mathrm{K} \mathrm{em}$ comparação ao Ca aos ligantes orgânicos quando encontrado simultaneamente no solo (ZIA et al., 1999). O terceiro e quarto componente principal explicaram menos de $10 \%$ da variância dos dados e contribuíram pouco para explicar a variabilidade dos atributos químicos e sua relação com os atributos do solo (Tabela 4) e do relevo (Tabela 5).

$\mathrm{Na}$ profundidade de $0,1-0,2 \mathrm{~m}$, no primeiro componente principal $\left(\mathrm{CP} 1_{0,1-0,2}\right)$, explicando $31,28 \%$ da variação total dos dados, observou-se forte correlação positiva com teores de $\mathrm{Al}$ no solo e negativa com $\mathrm{Ca}$ e $\mathrm{Mg}$ (Tabela 4). O CP1 $1_{0,1-0,2}$ pode ser interpretado novamente como resultante das reaçôes de neutralização da acidez do solo em função da aplicação de corretivos, porém não havendo nenhum outro atributo do solo (matéria orgânica e textura, por exemplo) inter-relacionado a esse grupo. $\mathrm{O}$ $\mathrm{CP} 2_{0,1-0,2}$, que participa com $15,2 \%$ da variação total, possui forte correlaçáo positiva da areia grossa, paralelamente, à correlação negativa do K e positiva do $\mathrm{P}$. Os componentes da matéria orgânica não tiveram correlações com $\mathrm{CP} 1_{0,0-0,1}$ e CP2 $2_{0,1-0,2}$ acima do critério de seleçáo adotado, provavelmente, devido à diminuição da contribuiçáo da matéria orgânica em profundidade. A correlação de Pearson entre $\mathrm{CTC}_{\text {efetiva }} \mathrm{x}$ argila de $0,45(\mathrm{p}<0,001)$ na profundidade de 0,1- 0,2 m está na faixa entre 0,4 e 0,6 considerada de intensidade moderada, conforme sugerido em SANTOS et al. (2012). Em contrapartida, a correlaçâo entre CTC $_{\text {efetiva }} \mathrm{x}$ COT é de 0,08 ( $p>0,05)$ nesta mesma profundidade.

A correlaçáo positiva entre $\mathrm{P}$ e $\mathrm{CP} 2_{0,1-0,2}$, sugere uma tendência de aumento da disponibilidade de P com maiores teores de areia grossa e diminuiçáo com maiores teores de argila, uma vez que sua retenção torna-se mais fraca e a disponibilização mais fácil com a diminuição do teor de argila (MACHADO et al., 2011). O terceiro e quarto componente principal explicaram menos de $10 \%$ da variação total dos dados, sendo tal variaçáo pouco relacionada com o relevo (Tabela 5).

Parte da variação dos atributos na $\mathrm{ACP}$ que tiveram maior correlação com o relevo (Tabela 5) na profundidade de $0,0-0,1 \mathrm{~m}$, pôde ser explicada em CP1 $1_{0,0-0,1}$ e CP2 ${ }_{0,0-0,1}$ (54\% de variância acumulada); na profundidade de 0,10,2 m pôde ser explicada em $\mathrm{CP} 2_{0,1-0,2}(15 \%$ da variância) (Tabela 4). Este fato implica que outras fontes de variação (atividade microbiana, densidade de plantas, manejo adubação entre outros), muitas vezes de difícil distinção no solo, controlam a parte da variabilidade dos atributos químicos no solo não relacionada com o relevo.

A análise geoestatística dos componentes principais foi realizada utilizando-se os escores atribuídos às amostras referentes aos dois primeiros componentes principais, que explicaram a maior percentagem da variação total dos

Tabela 5. Coeficiente de correlação de Pearson entre componentes principais e os atributos geomorfológicos altitude (ALT), fluxo acumulado do escoamento superficial (FAES) e declividade (DECL)

\begin{tabular}{|c|c|c|c|c|c|c|c|c|}
\hline & \multicolumn{4}{|c|}{ Profundidade $0,0-0,1 \mathrm{~m}$} & \multicolumn{4}{|c|}{ Profundidade $0,1-0,2 \mathrm{~m}$} \\
\hline & CP1 & CP2 & CP3 & CP4 & CP1 & CP2 & CP3 & CP4 \\
\hline ALT & $0,75^{* * *}$ & $-0,68^{* * *}$ & $-0,18^{\text {n.s }}$ & $0,00^{\text {n.s }}$ & $-0,09^{n . s}$ & $-0,57^{* * *}$ & $0,08^{\text {n.s }}$ & $-0,20^{*}$ \\
\hline FAES & $-0,28^{* *}$ & $0,51^{* * *}$ & $-0,09^{n . s}$ & $0,19^{n . s}$ & $0,12^{\text {n.s }}$ & $0,31^{* *}$ & $0,00^{\text {n.s }}$ & $0,11^{\text {n.s }}$ \\
\hline DECL & $-0,37^{* *}$ & $0,37^{* *}$ & $0,21^{*}$ & $-0,13^{\text {n.s }}$ & $0,02^{\text {n.s }}$ & $0,22 *$ & $0,05^{\text {n.s }}$ & $0,19^{\text {n.s }}$ \\
\hline
\end{tabular}

Correlaçấo de Pearson em que ${ }^{* * *}$ correspondem a $\mathrm{p}<0,001 \mathrm{e}^{* *}$ a $\mathrm{p}<0,01 \mathrm{e}^{\mathrm{n} . \mathrm{s}}$ corresponde a $\mathrm{p}>0,05$. 
dados (Tabela 6). Quanto maiores os escores atribuídos às amostras, maior sua contribuiçáo para a correlaçáo positivas entre as variáveis e os componentes principais enquanto menores escores significam maior contribuição para correlaçóes negativas. Para tanto, foram feitos ajustes desses escores a modelos teóricos de semivariogramas (Tabela 6).

Analisando espacialmente, o $\mathrm{CP} 1_{0,0-0,1}$ proporcionou maior dependência espacial (37\%), maior alcance (42 m) e padrão de distribuição espacial fortemente relacionado ao relevo (Tabela 6, Figura 3). Pode-se observar que no terço superior, o $\mathrm{CP} 1_{0,0-0,1}$ possui escores positivos, indicando que em maior altitude e menor declividade existe forte relaçáo com o grupo de variáveis argila, COT, Ca, $\mathrm{Mg}$, saturação por bases (V) e $\mathrm{pH}$. Essa relação foi constatada pela correlaçáo positiva entre $\mathrm{CP} 1_{0,0-0,1}$ e altitude, e negativa entre $C P 1_{0,0-0,1}$ e declividade (Tabela 5), o que indica forte influência de fatores intrínsecos (relevo) como geradores da variabilidade explicada pelo $\mathrm{CP} 1_{0,0-0,0)}$.

No CP1 $1_{0,1-0,2}$ ocorreram menores valores de alcance $(9,2 \mathrm{~m})$ e menor dependência espacial (59\%), indícios da influência de fatores extrínsecos ou menor correlação com atributos de natureza intrínseca (argila e areia grossa, por exemplo). Não foram notadas correlaçōes significativas entre a CP1 $1_{0,1-0,2}$ e atributos geomorfológicos (Tabela 5).
$\mathrm{Na}$ profundidade de $0,1-0,2 \mathrm{~m}$, em maiores altitude são também foram verificados os maiores teores de argila (dados não apresentados), porém não refletiu em melhor relaçáo da $\mathrm{CP} 1_{0,1-0,2}$, altitude e fraçóes granulométricas, como para $\mathrm{CP} 1_{0,0-0,1}$. Observa-se, dessa forma, que os primeiros componentes principais das duas profundidades $\left(\mathrm{CP} 1_{0,0-0,1}\right.$ e $\left.\mathrm{CP} 1_{0,1-0,2}\right)$ tiveram forte relação com bases e alumínio trocável, contudo o comportamento espacial desses atributos inter-relacionados é diferente entre as duas profundidades avaliadas (Tabelas 4 e 5).

Com relação ao $\mathrm{CP} 2$, em ambas as profundidades, fortemente correlacionado com a areia grossa, pode-se observar maior alcance como reflexo da contribuiçáo do $\mathrm{K}(39,7 \mathrm{~m})$ e da areia grossa, que é um atributo do solo de caráter intrínseco, e em geral, de maior alcance (Cambardella et al., 1994). Na CP2 $2_{0,0-0,1}$ e CP2 ${ }_{0,1-0,2}$, observa-se uma correlaçáo negativa do $\mathrm{K}$ e argila e positiva da areia grossa (Tabela 4). A CP2 $2_{0,0-0,1}$ e CP2 $2_{0,1-0,2}$ tiveram correlaçóes negativas com altitude e positivas com FAES e declive (Tabela 6).

Mesmo com a aplicação anual de $\mathrm{K}$ em toda a área estudada, via adubação, ele se redistribui mais facilmente na paisagem tanto pela maior mobilidade desse nutriente no solo como pela fraca relaçáo com a areia grossa, gerando

Tabela 6. Modelos teóricos e parâmetros estimados dos semivariogramas experimentais ajustados aos valores de escores dos dois primeiros componentes principais nas profundidades de 0,0-0,1 e 0,1-0,2 m e a estatística da validação cruzada

\begin{tabular}{|c|c|c|c|c|c|c|c|c|c|c|}
\hline \multirow{2}{*}{ Var. } & \multirow{2}{*}{$\begin{array}{l}\text { Profundidade } \\
\text { (m) }\end{array}$} & \multirow{2}{*}{ Modelo ${ }^{(1)}$} & \multicolumn{5}{|c|}{ Parâmetros ${ }^{(2)}$} & \multicolumn{3}{|c|}{ Validação Cruzada(4) } \\
\hline & & & $C_{0}$ & $C_{0}+C_{1}$ & $\mathrm{C}_{0} /\left(\mathrm{C}_{\mathrm{o}}+\mathrm{C}_{1}\right)^{(3)}$ & A & $\mathbf{R}^{2}$ & Variância & Média & $\mathbf{r}$ \\
\hline CP 1 & \multirow{2}{*}{$0,0-0,1$} & ESF & 0,40 & 1,09 & 37 & 42,5 & 0,94 & 1,05 & 0,001 & $0,57^{* *}$ \\
\hline CP 2 & & GAUS & 0,51 & 1,34 & 38 & 55,2 & 0,96 & 1,03 & 0,004 & $0,65^{* *}$ \\
\hline CP 1 & \multirow{2}{*}{$0,1-0,2$} & GAUS & 0,61 & 1,02 & 60 & 9,7 & 0,97 & 0,94 & 0,003 & $0,36^{* *}$ \\
\hline CP 2 & & ESF & 0,53 & 1,17 & 45 & 37,6 & 0,94 & 1,01 & 0,006 & $0,55^{* *}$ \\
\hline
\end{tabular}

Var:: Variável; ( $\left.{ }^{1}\right)$ GAUS: gaussiano; ESF: esférico. $\left({ }^{2}\right)$ Co: efeito pepita; $\mathrm{Co}+\mathrm{C} 1$ : patamar; A: alcance; $\mathrm{R}^{2}$ : coeficiente de determinaçăo do semivariograma. $\left({ }^{3}\right)$ grau de dependência espacial expressa em percentagem ( $<25 \%$ - forte dependência espacial, 25 a $75 \%$ - moderada dependência espacial e $>75 \%$ - fraca dependência espacial) (CAMBARDELLA et al., 1994). $\left({ }^{4}\right)$ Validaçáo cruzada feita com a média do erro reduzido e a variância do erro reduzido, e coeficiente de correlaçáo simples entre dados observados e estimado da validação cruzada, sendo ${ }^{* *}$ significando $\mathrm{p}<0,01$.

(a) ESCORES AMOSTRAS

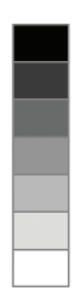

$-1,98$
$-1,46$
$-1,01$
$-0,58$
$-0,11$
0,28
0,56
1,01

(c) ESCORES AMOSTRAS
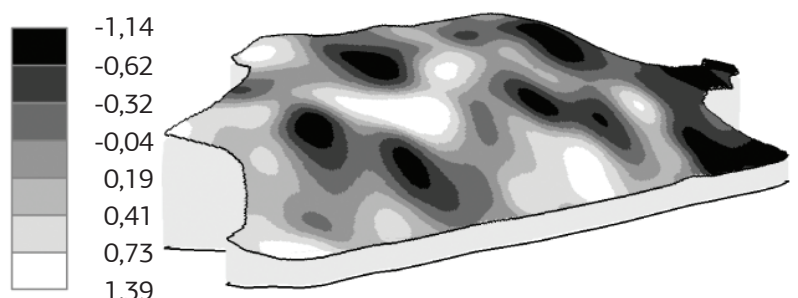

(b) ESCORES AMOSTRAS
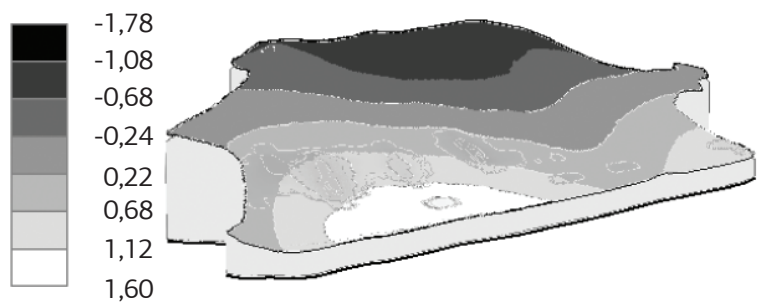

(d) ESCORES AMOSTRAS
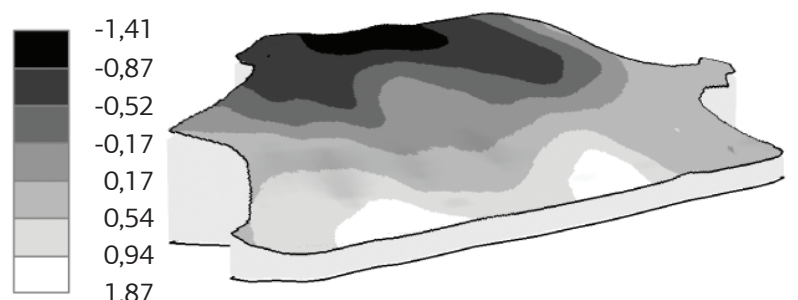

Figura 3. Distribuição espacial dos escores das amostras referentes ao CP1 (a) e CP2 (b) na profundidade de 0,0-0,1 m e das amostras referentes ao CP1 (c) e CP2 (d) na profundidade de 0,1-0,2 m. 
zonas homogêneas mais bem definidas, em comparação aos teores de Ca, Mg e P. SiLva et al. (2010) observaram correlaçáo negativa entre teor de $\mathrm{Ke}$ areia grossa em região cultivada sobre Latossolo. Sun et al. (2003) verificaram a diminuição do alcance e o aumento do efeito pepita em decorrência da adubação potássica em solo mais argiloso e de menor declividade.

A aplicaçáo de adubos e corretivos na área estudada é feita manualmente, aumentando a possibilidade de aplicação desuniforme e constituindo uma variabilidade oriunda do manejo (fator extrínseco). Parte dos nutrientes movimentados verticalmente (lixiviação) ocorre por vias que facilitam os fluxos preferenciais de água, tais como rachaduras, grandes canais e poros gerados pela biota e decomposição de material orgânico. Em subsuperfície, a influência do escoamento superficial na movimentação horizontal e redistribuiçấo dos nutrientes na paisagem é limitada, e o relevo tem menor relação com a distribuiçấo espacial dos atributos químicos e com os escores do $\mathrm{CP} 1_{0,1-0,2}$. Tal fato indica a importância de contextualização da profundidade e do grau de mobilidade dos nutrientes no solo para o estudo da variabilidade espacial e a estratificaçáo em zonas homogêneas baseadas no relevo.

O movimento horizontal de nutrientes via escoamento superficial é facilitado em maior declive, uma vez que a calagem e adubação são aplicadas sem incorporação ao solo, resultando em maior saturação de sítios de troca/adsorção em camadas superficiais, facilitando a presença desses nutrientes em solução e seu transporte via escoamento superficial. Segundo McDowell et al. (2001), a diminuição das doses aplicadas de nutrientes via adubação e calagem pode constituir fator importante para a menor perda de nutrientes pelo escoamento superficial e, de fato, os autores concluem que tal diminuição associada a práticas mecânicas são as mais eficientes. CANELlas et al. (2000), estudando uma sequência de solos em vertente de $500 \mathrm{~m}$ e declividade média de 5\%, constataram maiores teores de argila e carbono orgânico total em posições mais elevadas (terço superior), atribuindo às regiôes menos elevadas e de maior declive (terço médio) a dinâmica da água como responsável pela redistribuição de fraçôes mais solúveis (ácidos fúlvicos) da matéria orgânica. Ademais, a perda de partículas mais finas com tamanho das fraçôes argila, assim como a matéria orgânica mais solúvel durante o escoamento superficial em locais de maior declive, pode favorecer o transporte de nutrientes (WaLton et al., 2000).

A partir dos resultados, pode-se observar que as zonas homogêneas em relação aos teores de nutrientes no solo não dependem exclusivamente do relevo. Em maior altitude e declividade aproximadamente menor que $15 \%$, na profundidade de $0,0-0,1 \mathrm{~m}$, ocorre o maior enriquecimento da fração argila e, consequentemente de bases trocáveis $(\mathrm{Ca}, \mathrm{Mg}$ e $\mathrm{K})$, que por sua vez favoreceram os maiores valores de $\mathrm{pH}$ e menores teores de alumínio. Para o P, não foram evidentes zonas homogêneas passíveis de serem delimitadas em função do relevo e apesar do alto coeficiente de variação observado (Tabelas 1 e 2), pouco dessa variação foi controlada pelo relevo. Somente com o $\mathrm{K}$ nas duas profundidades, observaram-se zonas homogêneas semelhantes quanto aos seus teores devido à forte mobilidade em locais com maiores teores de areia e ao relevo ondulado favorável ao carreamento via escoamento superficial em locais de maior declividade.

\section{CONCLUSÃO}

Em relevo ondulado sob cafeeiro, a variabilidade espacial dos atributos químicos indica maior continuidade da dependência espacial na profundidade de $0,0-0,1 \mathrm{~m}$ onde existe maior influência do relevo e dos fluxos de água em superfície.

Com o uso da análise de componentes principais, atributos inter-relacionados às reaçôes ácido-base no solo ( $\mathrm{pH}, \mathrm{Ca}, \mathrm{Mg}, \mathrm{Al}, \mathrm{m}$ e $\mathrm{V}$ ) contribuem com o primeiro componente principal nas duas profundidades. A variabilidade espacial do primeiro componente principal na profundidade de $0,0-0,1 \mathrm{~m}$ é fortemente relacionada com o relevo (fator intrínseco) e teores de argila e areia grossa (atributos de caráter intrínseco). Em contrapartida, tal relação não é constatada na profundidade de $0,1-0,2 \mathrm{~m}$.

Zonas mais homogêneas em teores de nutrientes baseadas na relação com o relevo são mais bem evidenciadas para o K, nas duas profundidades estudadas $(0,0-0,1 \mathrm{e}$ $0,1-0,2 \mathrm{~m})$. Para os demais atributos químicos estudados, a separação de zonas homogêneas depende não somente do relevo, mas também da profundidade contextualizada.

\section{AGRADECIMENTOS}

À CAPES pelo auxílio financeiro junto ao Programa Nacional de Pós Doutorado/ PNPD e ao INCAPER pela concessáo da área de estudo.

\section{REFERÊNCIAS}

BORU゚VKA, L.; MLÁDKOVÁ, L.; PENÍŽEK, V.; DRÁBEK, O.; VAŠÁT, R. Forest soil acidification assessment using principal component analysis and geostatistics. Geoderma, v.140, p.374-382, 2007.

CAMBARDELLA, C.A.; MOORMAN, T.B.; NOVAK, J.M.; PARKIN, T.B.; KARLEN, D.L.; TURCO; R.F.; KONOPKA, A.E. Field-scale variability of soil properties in Central Iowa Soils. Soil Science Society of American Journal, v.58, p.1501-1511, 1994.

CANELLAS, L.P.; BERNER, P.G.; SILVA, S.G.; SILVA, M.B.; SANTOS, G.A. Fraçôes da matéria orgânica em seis solos de uma topossequência no Estado do Rio de Janeiro. Pesquisa Agropecuária Brasileira, v.35, p.133-143, 2000.

CARVALHO, M.P.; TAKEDA, E.Y.; FREDDI, O.S. Variabilidade espacial de atributos de um solo sob videira em Vitória Brasil (SP). Revista Brasileira de Ciência do Solo, v.27, p.695-703, 2003. 
CAVALCANTE, E.G.S.; ALVES, M.C.; SOUZA, Z.M.; PEREIRA, G.T. Variabilidade espacial de atributos químicos do solo sob diferentes usos e manejos. Revista Brasileira de Ciência do Solo, v.31, p.1329-1339, 2007.

CEDDIA, M.B.; VIEIRA, S.R.; VILLELA, A.L.O.; MOTA, L.S.; ANJOS, L.H.C.; CARVALHO, D.F. Topography and spatial variability of soil physical properties. Scientia Agricola, v.66, p.338-352, 2009.

CLAESSEN, M.E.C. (Org.). Manual de métodos de análise de solo. 2.ed. rev. atual. Rio de Janeiro: Embrapa-CNPS, 1997. 212p. (Embrapa - CNPS. Documentos, 1)

CRUZ, C.D.; REGAZZI, A.J. Modelos biométricos aplicados ao melhoramento genético. 2.ed. rev. Viçosa: UFV, 2001. 390p.

DOBBSS, L.; ALLEONI, L.R.F.; VELLOSO, A.C.X.; CANELLAS, L.P. Eletroquímica de Latossolos brasileiros após a remoção da matéria orgânica humificada solúvel. Revista Brasileira de Ciência do Solo, v. 32, p. 985-996, 2008.

FACCHINELLI, A.; SACCHI, E.; MALLEN, L. Multivariate statistical and GIS based approach to identify heavy metal sources in soils. Environmental Pollution, v.114, p. 313-324. 2001.

GAMMA DESIGN SOFTWARE. GS+: Geostatistics for environmental sciences. 7. ed. Michigan, Plainwell: Gamma Desing Software, 2004. 159p.

JAMES, F.C.; McCULLOCH, C.E. Multivariate analysis in ecology and systematics: Panacea or pandora's box? Annual Review of Ecology and Systematics, v.21, p.129-166, 1990.

MACHADO, V.J.; SOUZA, C.H.E.; ANDRADE, B.B.; LANA, R.M.Q; KORNDORFER, G.H. Curvas de disponibilidade de P em solos com diferentes texturas após aplicação de doses crescentes de fosfato monoamônico. Bioscience Journal, v.27, p.70, 2011.

McDOWELL, R.W.; SHARPLEY, A.N.; CONDRON, L.M.; HAYGARTH, P.M.; BROOKES, P.C. Processes controlling soil phosphorus release to runoff and implications for agricultural management. Nutrient Cycling in Agroecosystems, v.59, p.269-284, 2001.

OVALLES, F.A.; COLLINS, M.E. Variability of northwest Florida soils by principal component of analysis. Soil Science Society of American Journal, v.52, p.1430-1435, 1988.

PARTELLI, F.L.; VIEIRA, H.D.; SANTIAGO, A.R.; BARROSO, D.G. Produção e desenvolvimento radicular de plantas de café 'Conilon' propagadas por sementes e por estacas. Pesquisa Agropecuária Brasileira, v.41, p.949-954, 2006.

PREZOTTI, L.C.; GOMES, J.A.; DADALTO, G.G.; OLIVEIRA, J.A. Manual de Recomendação de Calagem e Adubação para o Estado do Espírito Santo. 5. a aproximação. Vitória: SEEA/ INCAPER/CEDAGRO, 2007. 305p.

SANTOS, D.S.; SOUZA, E.G.; NÓBREGA, L.H.P.; BAZZI, C.L.; GONÇALVES JÚNIOR, A.C. Variabilidade espacial de atributos físicos de um Latossolo Vermelho após cultivo de soja. Revista Brasileira de Engenharia Agrícola e Ambiental, v.16, p.843-848, 2012.

SILVA, S.A.; LIMA, J.S.S.; XAVIER, A.C.; TEIXEIRA, M.M. Variabilidade espacial de atributos químicos de um Latossolo vermelho-amarelo húmico cultivado com café. Revista Brasileira de Ciência do Solo, v.34, p.15-22, 2010.

SOUZA, Z.M.; MARQUES JÚNIOR, J.; PEREIRA, G.T.; BARBIERI, D.M. Small relief shape variations influence spatial variability of soil chemical attributes. Scientia Agricola, v.63, p.161-168, 2006.

SUN, B.; ZHOUB, S.; ZHAO, Q. Evaluation of spatial and temporal changes of soil quality based on geostatistical analysis in the hill region of subtropical China. Geoderma, v.115, p.85-99, 2003.

VIEIRA, S.R. Geoestatística em estudos de variabilidade espacial do solo. In: NOVAIS, R.F.; ALVAREZ, V.H.; SCHAEFER, G.R. (Ed.). Tópicos em Ciência do solo. Viçosa: Sociedade Brasileira de Ciência do Solo, v.1, 2000. p.1-54.

VIEIRA, S.R.; DECHEN, S.C.F.; SIQUEIRA, G.M.; DUFRANC, G. Variabilidade espacial de atributos físicos e químicos relacionados com o estado de agregação de dois Latossolos cultivados no sistema de semeadura direta. Bragantia, v.70, p.185-195, 2011.

WAIREGI, L.W.I.; van ASTEN, P.J.A. Norms for multivariate diagnosis of nutrient imbalance in arabica and robusta coffee in the east African highlands. Experimental Agriculture, v.48, p.448-460, 2012.

WALTON, R.S.; VOLKER, R.E.; BRISTOW, K.L.; SMETTEM, K.R.J. Experimental examination of solute transport by surface runoff from low-angle slopes. Journal of Hydrology, v.233, p.19-36, 2000.

WEBSTER, R., OLIVER M.A. Geostatistics for Environmental Scientists. 2.ed. Wiley, Chichester, 325p. 2007.

YEOMANS, J.C.; BREMNER, J.M.. A rapid and precise method for routine determimation of organic carbon in soil. Communications in Soil Science and Plant Analysis, v.19, p.1467-1476, 1988.

ZHANG, C.S.; McGRATH, D. Geostatistical and GIS analyses on soil organic carbon concentrations in grassland of southeastern Ireland from two different periods. Geoderma, v.119, p.261-275. 2004.

ZIA, K.M.; NAWAZ, H.; MURTAZA, G. Organic matter and $\mathrm{pH}$ effects on base Exchange in coarse textured soils. International Journal of Agriculture and Biology, v.1, p.36-38, 1999. 